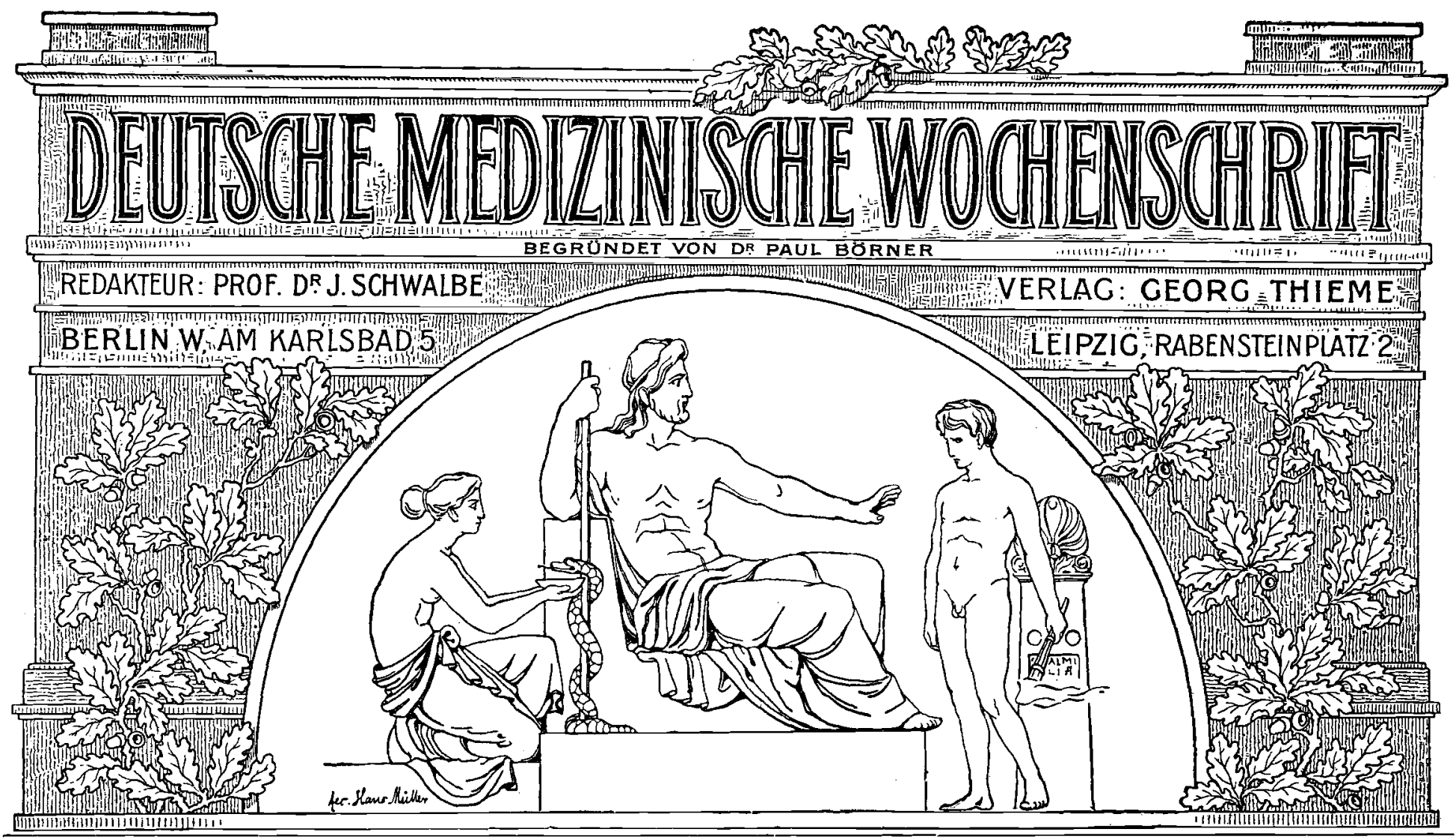

No. 43.

Berlin, den 22. Oktober 1908.

34. Jahrgang.

Aus der Universitäts-Frauenklinik in Greifswald.

\section{Prognose und Behandlung der puerperalen Infektion ${ }^{1}$ ).}

Von Prof. Dr. Max Henkel.

Es liegt auf der Hand, daß die Prognose der puerperalen Infektion eng verbunden ist mit der Aetiologie und der exakten Diagnose; und ich glaube, dab wir dank der unermüdlichen Studien nach der Richtung hin ein gut Stück in der Beurteilung des Kindbettfiebers vorwärts gekommen sind. Es ist nicht meine Aufgabe, meine Herren, Ihnen heute längst und allgemein Bekanntes zu wiederholen. Nur das, was meines Erachtens prinzipiell wichtig ist, möchte ich Ihnen in gedrängter Kürze vortragen, hier Glied an Glied reihen, sodaß ein möglichst einheitliches Ganzes entsteht, das bindende Schlüsse in bezug auf die Prognose zuläßt.

In Vordergrunde aller unserer Studien und Bemühungen nach der Aetiologie des Kindbettfiebers stehen selbstverständlich bakteriologische Untersuchungen. Hier lautet die Fragestellung ganz allgemein so: Existiert ein bestimmter Streptococcus, der das puerperale Fieber gant speziell hervorruft, oder handelt es sich nur um Streptococcen, die nur unter gewissen Voraussetzungen, sonst aber völlig identisch mit allen andern Streptococcen, das Kindbettfieber hervorrufen? Der zweite Teil der Fragestellung lautet so und ist zum Teil abhängig von der ersten: Wie kommtes, dab ein beliebiger Streptococcus die maligne Eigenschaft entfalten kann, die das Bild der puerperalen Infektion mit allen seinen Varianten erzeugt? Wir sehen also, dal die Beantwortung nicht ganz einfach ist; und sie ist gewif nicht damit erledigt, daß man das Vorhandensein von Streptococcen im Sekret der Scheide oder des Uterus einer Wöchnerin nachweist und damit Aetiologie und Prognose des Kindbettfiebers festlegt.

Wie übereinstimmend alle Untersucher aus den letzten Jahren angeben, finden sich im Sekret von Schwangeren und Wöchnerinnen Streptococcen, und zwar in einer Regelmäßig. keit, die jedes zufällige Auftreten, jeden Fehler etwa in der Untersuchungstechnik, ausschliebt. Von den wichtigeren hierher gehörenden Arbeiten möchte ich nennen diejenige von Natvig, Schenk und Scheib, Fromme, Kownatzky,

1) Nach einem Vortrage, gehalten auf der Naturforscherversammlung in Köln.
Schottmüller und andere. Auch meine eigenen Untersuchungen an nicht untersuchten Schwangern, die ich gemeinsam mit Bohn e vorgenommen habe und die von diesem in seiner Dissertation (Berlin 1902) niedergelegt sind, gehören hierher. Desgleichen meine Untersuchungen über den Bakteriengehalt des Lochialsekretes solcher Wöchnerinnen, die während der Geburt entweder überhaupt nicht untersucht wurden, dann nach stattgehabter innerer Untersuchung und schlieflich auch nach vorgenommener entbindender Operation. Die Resultate dieser Untersuchungen erscheinen demnächst in der Zeitschrift für Geburtshilfe und Gynäkologie.

Was die erste Arbeit betrifft, so untersuchten wir damals bakteriologisch das Scheidensekret von zehn nicht explorierten Schwangeren. Es handelte sich dabei vorwiegend um Frauen, die in der ersten Schwangerschaft standen und bei denen eine innere Untersuchung überhaupt nie vorgenommen war und die nie krank gewesen waren, namentlich nicht unterleibskrank. Unter den untersuchten Frauen befindet sich nur eine Zweitgebärende, sonst handelt es sich um Erstgebärende. Und von den Erstgebärenden war auch nur eine einzige, die einmal, und zwar längere Zeit (8 Wochen) vor der Sekretentnahme untersucht worden. Das damals erhobene Untersuchungsresultat gipfelte darin, daf die Scheide, auch der nicht Untersuchten, durchaus nicht als keimfrei anzusehen ist, sondern daß es jedesmal gelang, eine ganze Reihe der verschiedenartigsten Mikroorganismen aus dem Scheidensekret zu züchten. Fünfmal, also in der Hälfte der Fälle, konnten wir Streptococcen nachweisen, darunter zweimal den obligat-anaëroben Streptococcus, der seinerzeit von Menge-Krönig beschrieben wurde. Wir haben damals eine große Anzahl Ausstrichpräparate von dem Lochialsekret angefertigt und versucht, alle die verschiedenen Mikroorganismen, die wir im bakterioskopischen Bilde sahen, durch Kulturverfahren rein darzustellen. Nicht immer gelang dies, indem trotz der verschiedenartigsten Nährböden und Kulturverfahren wiederholt Bakterien nicht zu züchten waren, deren Vorhandensein aber durch das Ausstrichpräparat erwiesen war. Als Grund hierfür nahmen wir an, daf die Bakterien entweder an sich nicht fortpflanzungsfähig waren infolge mangelhafter Entwicklung, daß sie abgestorben waren, oder daß ihnen der gewählte Nährboden nicht zusagte.

Was nun speziell die Streptococcen anbelangt, so geht aus dieser Arbeit zur Evidenz hervor, daß sie keineswegs einen seltenen Befund in dem Sekret von Schwangeren darstellen. 
Auch das von Döderlein als normal bezeichnete Sekret, das die Konsistenz geronnener Milch hat und von saurer Reaktion ist, erwies sich nicht als keimfrei.

So kann es uns nicht wundern, wenn schon im Sekret gesunder Schwangerer, die nicht untersucht sind und bej denen auch jede gonorrhoische Infektion von vornherein auszuschlieken war, sich so häufig Streptococcen nachweisen lassen, daf wir dann auch in dem Sekret von normalen, selbst bei der Geburt nicht untersuchten Wöchnerinnen wieder auf Streptococcen treffen, wieder nachweisen können, daß die verschiedensten Mikroorganismen in der Scheide vorhanden sind.

Daraus folgt meines Erachtens zur Evidenz, daf die Anwesenheit der Streptococcen nicht allein genügt, um Kindbettfieber hervorzurufen, sondern daf zu dem Zweck noch andere Momente im Spiel sind, denen auf den Grund zu gehen jetzt unsere Aufgabe sein wird. Es wäre ja nun die einfachste Erklärung die, dal der Streptococcus, der bei der puerperalen Infektion in Frage kommt, ein ganz spezifischer Mikroorganismus ist. Aber alle die Untersucher, die sich mit dieser Frage beschäftigt haben, vermochten keine Anhaltspunkte ausfindig zu machen, die es uns mit Sicherheit ermöglichen, einen bestimmten Streptococcus des Puerperalfiebers zu unterscheiden. Je mehr wir uns mit dem Studium der Streptococcen beschäftigen, um so schwieriger scheint die Deutung der Befunde zu werden, die wir bei den einzelnen Streptococcen erheben. Aber schließlich und in letzter Linie kommen, wie mir scheinen will, die Unterschiede immer wieder auf die Kulturbedingungen hinaus; unter welchen äußeren Bedingungen resp. auf welchem Nährboden der Streptococcus wächst. Nur einen Streptococcus, glaube ich, müssen wir scharf trennen von allen übrigen, und das ist der obligat-anaërobe Streptococcus von KrönigMenge, der pathogene Eigenschaften aber nicht besitzt.

Während wir sonst durch geeignete Nährböden die Streptococcen verschiedener Provenienz kulturell einander nähern konnten, sodaß es unmöglich wurde, sie später zu differenzieren resp zu unterscheiden, gelang es uns mit dem anaëroben Streptococcus nicht; wir konnten ihn z. B. trotz aller Experimente nicht zu aërobem Wachstum bringen. Weitere Versuche ergaben, daß dieser Streptococcus nicht tierpathogen ist, sodaß er - auch nach den Beobachtungen am Menschen - wohl nur als ein harmloser Saprophyt aufgefaßt werden darf, der da gedeiht, wo durch das Wachstum anderer Mikroorganismen (wir fanden ihn nie isoliert) ein völliger Verbrauch von Sauerstoff erzielt wird. Auch im venösen Blut des Tierkörpers war er nicht weiter zu züchten, sodab der Sauerstoff, der im venösen Blut noch etwa $16 \%$ beträgt, von vornherein genügt, diesen Streptococcus zum Absterben zu bringen.

Wenn somit auch nachgewiesen ist (Zangemeister und Meissl, dal ein Artunterschied der Streptococcen nicht besteht, so müssen wir daran festhalten, daß die Wirksamkeit der Streptococcen selbst bedingt wird durch äufere Momente, die abhängig sind von den Bedingungen, unter denen sie gedeihen. Hinsichtlich der Virulenz können die größten Unterschiede bestehen, die sich durch das Kultur verfahren - wenn überhaupt - immer nur in der ersten Zeit ihrer künstlichen Züchtung werden kontrollieren lassen.

Zur Differenzierung der Streptococcen ist in letzter Zeit das Phänomen der Hämolyse herangezogen worden. Arbeiten, die sich damit beschäftigen, gibt es eine ganze Reihe, aber die Befunde weichen zum Teil stark auseinander. Die Frage der Hämolyse ist durch Bordet ins Rollen gebracht und dann von Schottmüller weiter zur Differenzierung einzelner Streptococcenarten benutzt worden. Auf Grund des Kulturverfahrens und der Bildung eines hämolytischen Hofes unterschied dieser letzte Autor drei große Arten von Streptococcen, von denen der eine, Streptococcus longus pathogenes, auf Blutagar kleine Kolonien mit einem wasserhellen Hof bildet; die zweite Art, Streptococcus mitior senviridans, bildet auf Blutagar grünliche Kolonien ohne das Phänomen der Hämolyse. Als dritte Art bezeichnet Schottmüller den Streptococcus mucosus, der auf Blutagar schleimige Kolonien ohne Hämolyse bildet. Es unterliegt keinem Zweifel, daf die Beobachtungen Schottmüllers zu Recht bestehen, sowohl hinsichtlich ihres Vorhandenseins wie ihrer Deutung; einmal spricht dafür die Kritik des Untersuchers selbst und zweitells das große Material, das jeden Irrtum ausschliebt. Die Frage ist nur die, ob die von Schottmüller beobachteten Tatsachell genügen, um bindende Schlüsse für die Unterscheidung und die Pathogenität der einzelnen Streptococcenarten zu ziehen. Durch zahlreiche Arbeiten sind wir heute mit Sicherheit darüber orientiert, daß ein Unterschied in der Art der Streptococcen, mögen sie rein saprophytärer Herkunft sein oder auf der anderen Seite der Erreger schwerer septischer Erkrankung gewesen sein, nicht besteht. An der Arteinheit der Streptococcen, abgesehen von dem obligat anaëroben, über den ich vorher berichtete. müssen wir also festhalten. Die Entscheidung der Frage, inwieweit das Phänomen der Hämolyse zur Beurteilung der Frage nach der Malignität der Streptococcen zu verwerten wäre, hat ebenfalls verschiedene Bearbeiter gefunden. Die ausgedehntesten Untersuchungen hat wohl Fromine nach der Richtung hin angestellt. Sein Urteil läßt sich dahin zusammenfassen. daß der Nachweis der hämolysierenden Streptococcen ein sicheres Zeichen einer bestehenden puerperalen Infektion sei. In einem Aufsatz über die Hämolyse der Streptococcen (Berliner klinische Wochenschrift 1908, No. 19) schliebt er sich der Veitschen Ansicht an, daß Puerperalfieberkranke mit typisch hämolysierenden Streptococcen in dem Lochialsekret eine Gefahr für ihre Umgebung bilden. Und in einer anderen Arbeit: Klinische und bakteriologische Studien zum Puerperalfieber legt Fromme auch wieder einen außerordentlichen Wert auf den Nachweis hämolysierender Streptococcen in dem Uterus oder der Scheide fiebernder Wöchnerinnen. Hier aber geht er noch einen Schritt weiter in bezug auf die Prognosestellung des Falles, indem er sagt: „Erscheinen die Keime in größeren Mengen im Blute, sind sie darin wiederholt nachweisbar, so wird die Prognose noch trüber.“

Fromme hat sich also auf Grund seiner Untersuchungen den Schottmüllerschen Beobachtungen angeschlossen und bestätigt, daß es durch das Phänomen der Hämolyse gelingt, abgesehen von der Prognosenstellung des Falles, einen hoch pathogenen, das Puerperalfieber verursachenden Streptococcus von den übrigen nur wenig oder garnicht pathogenen Streptococcen auf das genaueste zu unterscheiden.

Nicht zu diesem Resultat, sondern zu einem ziemlich abweichenden ist Freymuth gekommen, der dem Phänomen der Hämolyse nur eine sehr untergeordnete Bedeutung beisprechen zu mïssen glaubt. Mit dieser Arbeit von Freymuth hat sich Fromme selbst (1. c.) abgefunden, und zwar damit, daf er, wie mir scheint mit Recht, Freymuth den Vorwurf macht, nicht gleichwertiges Material benutzt zu haben, um zu einer so wichtigen Frage Stellung nehmen zu können. Während Schottmüller und Fromme nur frisch vom Menschen gezüchtete Streptococcen benutzten und dem Phänomen der Hämolyse Bedeutung zuerkannten im Einklang mit den vorhandenen klinischen Erscheinungen, wurden auf der anderen Seite ausgedehnte Kulturversuche mit wenigen, lange Zeit weiter gezüchteten Streptococcen-Stämmen vorgenommen. Natwig und Schlesinger vermochten die Hämolyse einem Stamm anzuzüchten. Auch Heynemann ist es gelungen, einem saprophytischen Streptococcus hämolysierende Wirkung aufzuzwingen. Notwendig ist dazu die Tierpassage; ohne dieselbe ist es bisher keinem Untersucher gelungen, an sich nicht hämolysierende Streptococcen dazu zu bringen.

Es darf uns diese Tatsache auch nicht überraschen, denn ich weiß nach meinen eigenen Erfahrungen, daß das Phänomen der Hämolyse an gewisse Umstände gebunden ist, und zwar dokumentiert sich das in der Weise, daß ein sicher hämolysierender Streptococcus, frisch aus dem Menschenblut mit diesem Phänomen gezogen, schon nach wenigen Uebertragungen diese Eigenschaft verlor, während bei anderen die hämolysierende Wirksamkeit auch trotz der fortgesetzlen Kulturübertragung lange Zeit deutlich ausgesprochen erhalten blieb. Auch die Unterschiede in dem zeitlichen Auftreten der Hämolyse und die verschiedene Ausdehnung der hämolytischen Zone deuten auf gewisse Unterschiede hin. Und auf der anderen Seite müssen wir mit der Tatsache rechnen, daß der puerperal-pathogene Streptococcus, der in einwandfreier Weise 
auf der Blutagarplatte das Phänomen der Hämolyse bildet, von vornherein nicht diese Eigenschaft besessen hat, sondern dâ er diese den besonderen Umständen, durch die er gleichzeitig für Menschen oder Tiere pathogen wird, verdankt. Der Blutnährboden allein kann die Frage der Hämolyse und die Bedeutung des Phänomens nicht entscheiden. Und darum schließe ich mich den Ansichten Frommes durchaus an, wenn er die Einwände, die erhoben worden sind, zurückweist. In gleichem Sinne halte ich es für ungerechtfertigt, wenn Beitzke auf Grund von Kulturstudien, die er mit 30 Stämmen von Streptococcen unter Benutzung von Blutnährböden gemacht hat, angibt, daß dieses Verfahren keine Differenzierung der Streptococcen in mehrere Unterarten im Sinne Schottmüllers ermöglicht.

Ich möchte nicht falsch verstanden werden nach der Richtung hin, daß ich mich etwa Schottmüller in der Einteilung der Streptococcen in die Unterarten, wie er sie gibt, anschliefe. Ich bin vielmehr der Ansicht, daß auch ihm eine Art Selbsttäuschung hierin untergelaufen ist, daß es sich in Wirklichkeit trotz der verlockenden Kulturergebnisse nicht um verschiedene Arten von Streptococcen handelt, sondern nur um gewisse Unterschiede, die sich aus der verschieden. artigen Provenienz etc. erklären lassen. Bezüglich der Deutung der Hämolyse bei Streptococcen, die frisch aus dem Menschenblut kommen und mit diesem gezüchtet sind, bin ich dagegen, so weit meine Erfahrungen reichen, durchaus der Ansicht von Schottmüller und Fromme.

Was nun die speziell prognostische Verwertung des $\mathrm{Ph}$ än omens der Hämoly se anbelangt, so liegen meines Erachtens nach meinen eigenen zahlreichen Untersuchungen und bakteriologischen Erfahrungen, die ich mir durch das Studium der Streptococcen aus der Scheide, dem Uterus, dem Blut und Harn von Schwangern und Wöchnerinnen erworben habe, die Dinge nicht so, dab unter allen Umständen das Auftreten der Hämolyse als ein Symptom zu betrachten ist, das allein und ganz allgemein die Prognose des Falles zu einer unbedingt schlechten macht. Auf die Begründung dieses Urteils werde ich weiter unten eingehen, indem ich an der Hand eines ganz charakteristischen und in einwandfreier Weise untersuchten Falles in der Lage bin nachzuweisen, daß die Hämolyse auch bei der Kultur von Streptococcen beobachtet werden kann, wo keineswegs eine schwere puerperale Infektion vorliegt. So weit ich jetzt imstande bin, über die Dinge ein Urteil abzugeben, würde ich es dahin zusammenfassen, daß, wenn in dem Blute einer fiebernden Wöchnerin Streptococcen mi deutlicher hämolytischer Wirkung auftreten, dieser Befund unter allen Umständen eine ernste Berücksichtigung beansprucht, und zwar einmal durch das Auftreten der Streptococcen im Blute überhaupt und zweitens durch die Entfaltung der Hämolyse. $\mathrm{Zu}$ dem Auftreten der Streptococcen im Blute ist nun zu bemerken, daß ihr Vorhandensein in demselben an sich noch nichts unbedingt Ungünstiges, was für den sicher letal endenden Verlauf der Infektion spricht, bedeutet.

Jeder, der sich mit der Bakteriologie des Blutes beschäftigt hat, kennt die Fälle, in denen Streptococcen im Blute auftreten und bei denen die Infektion doch einen verhältnismä日ig günstigen, schnell zur Heilung führenden Verlauf genommen hat. Soll man aus dem bakteriologischen Blutbilde etwas Bestimmtes aussagen, so ist einmal entscheidend der Zeitpunkt des Auftretens der Bakterien im Blute nach der mutmaflich stattgehabten Infektion - in unserem Fall also nach der Entbindung -, zweitens die Zahl der angegangenen Kolonien und drittens die Schnelligkeit der Entwicklung derselben, auch bezüglich der Hämolyse. Und ich glaube mit aller Sicherheit aussprechen zu dürfen, daß die Prognose absolut infaust ist, wenn es gelingt, schon am nächsten oder übernächsten Tage nach der Entbindung in der Blutagarplatte zahlreiche Kolonien von Streptococcen mit hämolytischem Hof nachzuweisen, die schon deutlich nach 9-12 Stunden dauernder Bebrütung der Platte mit dem bloßen Auge zu erkennen sind. Ergibt sich nun nach einer weiteren Blutuntersuchung (etwa 24 Stunden nach der ersten), dal noch eine weitere Vermehrung der Streptococcen eingetreten ist, so halte ich die Prognose des Falles für absolut infaust,
Es wird die Aufgabe weiterer Untersuchungen sein festzustellen, ob bei den Streptococcen-Infektionen des Puerperalfiebers die Hämolyse eine häufigere Begleiterscheinung des Wachstums der Streptococcen ist als bei Infektionen, die unter anderen Voraussetzungen zu einem Uebertritt der Streptococcen in das Blut führen. Jedenfalls habe ich bei meinen Blutuntersuchungen auch Fälle beobachtet, wie folgenden:

58 jährige Frau wird wegen Prolaps operiert, und zwar handelt es sich um eine einfache Laws on-T aitsche Operation, die von einem meiner Assistenten ausgeführt wurde. Sehr bald danach traten die Symptome einer Peritonitis auf, an die sich unter dem Bilde allgemeiner Sepsis der Tod sechs Tage nach der Operation anschloB. Durch die Autopsie, die bei dem Interesse, das wir allen Infektionsfällen unserer Klinik entgegenbringen, in liebenswürdiger Weise von Herrn Geheimrat Grawitz, dem Direktor des hiesigen Pathologischen Institutes, selbst ausgeführt wurde, ergab sich, daß die Operationswunde nicht den Ausgang der Infektion darstellte, sondern daß hierfür eine doppelseitige alte und sehr kleine Pyosalpinx beschuldigt werden mußte, deren Keime durch das Trauma der Operation offenbar wieder mobil gemacht worden waren und die dann zu der allgemeinen Sepsis führten.

In diesem Falle ergab die Blutuntersuchung schon am dritten Tage post operationem bei einer verhältnismäßig niedrigen Temperatur eine massen hafte Ueberschwemmung des Blutes mit Streptococcen, und zwar ohne daß auch nur die geringste Hämolyse weder in der übrigen Zeit der Beobachtung der Blutagarplatte noch auch nach mehreren Tagen zu konstatieren gewesen wäre. Es ist also schon möglich, daß die Hämolyse, da wir diese bei den schweren Puerperalfieberfällen so besonders häufig beobachten, vielleicht doch durch die andersartige Zusammensetzung des Blutes der Wöchnerin unterstützt wird. Worauf in letzter Linie das Phänomen der Hämolyse beruht, ist uns also bislang noch nicht gegenwärtig.

Wir wissen durch die Untersuchungen von Schottmüller und Lenhartz, daß durch die Menge des Blutes, die wir dem Agar zusetzen, die Hämolyse deutlich oder weniger deutlich, früher oder später hervorgerufen werden kann. Und die Mischung $2 \mathrm{ccm}$ Blut mit $5 \mathrm{ccm}$ Agar, wie sie Schottmüller schlieblich angegeben hat, wird jeder Untersucher als bestes Verhältnis bestätigen können. Aber abgesehen von der Menge des Blutes sprechen auch unzweifelhaft noch andere Dinge ein wichtiges Wort, über die Sicheres auszusagen ich nicht in der Lage bin. Jedenfalls steht die Tatsache fest, wenn man mit einem hämolysierenden Streptococcus experimentiert und einmal ihn mit Agar und dem Blut seiner Trägerin mischt und auf der anderen Seite ihn mit Blut einer anderen Frau und ebenfalls Agar in demselben Prozentsatz mischt, daß dann die Hämolyse in ihrem Auftreten und in ihrer Ausdehnung nicht die gleiche ist. Und zwar hat sich hier die Tatsache ergeben, dab bei einem Streptococcus, aus dem Uterussekret rein gezüchtet und im Blutagargemisch kultiviert, die Hämolyse in dem Falle intensiver war, in dem das Blut von der Patientin stammte, aus deren Uterussekret der Streptococcus gezogen war; mit dem Blut einer anderen Frau trat die Hämolyse etwas später ein, und es erreichte der g]ashelle Hof auch nicht die Ausdehnung.

Ich glaube nicht, daß man hieraus einen Schlus ziehen kann dahingehend, daß dieses Vorkommen gegen den Wert der Hämolyse spräche, aber immerhin schien es mir interessant genug, um es zu erwähnen. Weiter - diese Versuche sind noch nicht abgeschlossen - scheint es möglich zu sein, dal der Sauerstoffgehalt des Blutes ebenfalls einen gewissen Einfluß auf das Zustandekommen der Hämolyse ausübt und daf mit der Abgabe des Sauerstoffes auch die Hämolyse geringer wird. Aber, wie gesagt, ich deute diese Beobachtung nur an, um zu zeigen, daß wir bei dem Studium über die Pathogenität der Streptococcen mit der einfachen Konstatierung des Auftretens der Hämolyse noch nicht am Ende unserer Studien sind. Von besonderer Bedeutung scheint mir das frühzeitige Auftreten der Hämolyse in der Kultur, etwa neun Stunden nach der Bebrütung, und der Nachweis zahlreicher Streptococcen im Blut zu sein.

Das Vorhandensein der Streptococcen im Uterussekret selbst, mit gleichzeitiger hämolytischer Wirkung, beansprucht hinsichtlich der Prognose des Falles bei weitem nicht den Wert 
und die Bedeutung der Blutuntersuchung, wie mir zahlreiche Untersuchungen bestätigt haben.

Es bleibt noch zu unterscheiden, welcher Wert den aus dem Urin gezogenen Streptococcen fiebernder Wöchnerinnen zuzusprechen ist. Auch hierüber sind Untersuchungen an meiner Klinik im Gange, über die ich an anderen Stellen berichten werde. Der Zusammenhang mit der puerperalen Infektion scheint mir ein ganz ungezwungener zu sein, analog deln Auftreten der Bazillen im Urin Typhuskranker. Ich habe seinerzeit als Assistent der Inneren Abteilung des Eppendorfer Krankenhauses (Oberarzt Dr. Ru m pel) Typhuspatientinnen in der Weise untersucht, daB zur Stellung resp. bakteriologischen $\mathrm{Be}$ stätigung der Diagnose Kulturen aus Blut und Urin angelegt wurden. Wiederholt ließ sich dann die Tatsache feststellen, die auch andere Untersucher erheben konnten, dab es gelingt, im Urin die Typhusbazillen nachzuweisen, und zwar manchmal zu einer Zeit, wo die Blutuntersuchung noch nichts Positives ergeben hatte. Während nun das Auftreten von Typhusbazillen im Urin, ohne einem Irrtum zu erliegen, wohl so erklärt werden mub, daB die Bakterien vom Darm resorbiert in die Blutbahn gelangt sind und dann mit dem Harn ausgeschieden werden, ist diese Deutung in analoger Weise für das Puerperalfieber nicht ohne weiteres zulässig, denn man kann ja auch annehmen, daß bei den innigen Beziehungen zwischen Blase und dem weiblichen Genitale auf dem Wege der zahlreich vorhandenen Lymphbahnen die Streptococcen durch die Blasenwand in die Blase hineingelangen. Bei der Häufigkeit, mit der man bei eitrigen Entzündungen im kleinen Becken fiebernder Wöchnerinnen im Urin Streptococcen findet, ist jedenfalls diese Delltung des Hineingelangens der Streptococcen in die Blase für wahrscheinlich zu halten. Eine weitere Erklärung für das Auftreten von Streptococcen im Urin, namentlich bei puerperaler Pyämie, bieten die metastatischen Abszesse in der Niere. Je nach den Beziehungen dieser Abszesse zu den Nierenbecken wäre das Auftreten der Streptococcen im Urin nicht besonders bemerkenswert. Während aber in diesen Fällen die Infektion eine absteigende $\mathrm{zu}$ nennen ist (Erkrankung der Niere, des Nierenbeckens und dann der Blase), kommt es bei den erst angedeuteten Fällen, besonders wenn eine Urinstauung in der Blase sich entwickelt, vor, daß erst sekundär die Infektion des Nierenbeckens und der Niere entsteht. Diese Beteiligung der Harnwege im Wochenbett und das Auftreten der Streptococcen an dieser Stelle des Organismus verdient meines Erachtens eine noch viel größere Berücksichtigung, als ihr im allgemeinen zurzeit eingeräumt wird. So gehört denn zu einer regelrechten bakteriologischen Untersuchung von Puerperalfieber die des Blutes, des Urins und die Untersuchung des Uterussekrets. Ueber die Technik der Entnahnie des Blutes und des Urins will ich das hervorheben, dab ich mich niemals darauf beschränke, das Blut durch Einstich mit der Nadel in die Vene und Ansaugen mit der Spritze zu entnehmen. Dieses Verfahren scheint mir deswegen nicht sicher genug, weil tiefer in der Haut gelegene Keime leicht einmal eine Täuschung hervorrufen können. Aus dem Grunde wird bei mir in jedem Fall die Vene freigelegt, die Kanüle in die Vene eingeschoben und dann das Blut aufgefangen.

Die Urinuntersuchung wird in der Weise gemacht, daß die Harnröhrenmündung gereinigt, in die Blase ein steriler Glaskatheter eingeführt wird, durch den zunächst ein Teil des Urins wegläuft. Erst der Rest des Urins (man kann es an dem Druck, unter dem der Urin abläuft, leicht erkennen) wird in einem sterilen Kolben aufgefangen. Je nachdem der Urin sauer oder alkalisch ist, wird ein saurer Nährboden (Bouillon, Agar) zur Anlage der Kultur genommen und bei trübem Urin eine Oese, bei klarem Urin einige Kubikzentimeter in den Nährboden übertragen.

Bezüglich der Sekretentnahme des Uterus bin ich auf Grund ans. gedehnter Vergleiche zu dem Resultat gekommen, daß es nicht nötig ist, ein Röhrchen in den Uterus einzuführen (D öderle in, Wal thhard), sondern es genügt vollkommen, wenn man mit sterilem Speculum (zwei Simonsche Platten) die Portio einstellt, die Muttermundslippen mit geeigneten Zangen, die keine Stichverletzungen machen, wie z. B. die Kugelzangen, die vielmehr an der Stelle der Spitze eine kleine Platte haben, auseinandernimmt, mit sterilen Tupfern das anhaftende Sekret gründlich abwischt und dann mit der Platinöse das Sekret aus dem Uterus entnimmt. Es genügt dieses Verfahren vollständig zur Beantwortung der uns interessierenden Frage.
Die Gründe, die mich veranlaßt haben, die Untersuchungstechnik nach der angegebenen Richtung zu verändern, bestehen darin, daß zuerst und als wichtigstes Ergebnis die Befunde keinen nennenswerten Unterschied im Verhältnis zu der früheren Technik (Entnahme durch das Röhrchen) ergaben. Sodann haftet diesem einfachen Verfahren eine Reihe von Vorteilen an, die die ganze Untersuchung wesentlich vereinfachen und ihre Gefahr für die fiebernde Wöchnerin herabsetzen. Wiederholt habe ich es gesehen, daß nach der Sekretentnahme mit dem Röhrchen, zumal wenn die Frau unruhig war und Verletzungen des Endometriums sich nicht vermeiden ließen, daß dann eine wesentliche Verschlimmeruug des Zustandes der Frau als Folge der Untersuchung eintrat. Und das läßt sich unmöglich mit dem Zweck der Untersuchung vereinbaren. Weiter glaube ich auch, daß man mit der kleinen, gut gebogenen Platinnadel leichter Sekret aus dem Uterus bekommt, es ist ja nur wenig Material nötig, und drittens glaube ich heute auf Grund meiner Erfahrungen, daß dem Resultat der bakteriologischen Untersuchung des Endometriums bei weitem nicht mehr die Bedeutung zuzusprechen ist, wie es bislang allgemein angenommen wird. Ich habe zu viel Fälle von Streptococcen im Sekret fiebernder und nicht fiebernder Wöchnerinnen gefunden, ohne daß deswegen die Prognose des Falles eine ungünstige gewesen wäre, sodaß ich mich nicht mehr zu denjenigen Autoren rechnen kann, die auf Grund im Uterus vorhandener Streptococcen bindende Schlüsse für die Prognose des Falles ziehen.

Ich glaube auch, dal meine Zweifel bezüglich des therapeutischen Effektes berechtigt sind, die ich denjenigen Untersuchern entgegenbringe, die bei fiebernden Wöchnerinnen im Uterussekret Streptococcen nachgewiesen haben, lediglich daraufhin ein entsprechendes Serum verabfolgt haben und dann konstatieren konnten, daß früher oder später nach Einverleibung des Serums eine Besserung eintrat. Derartige Fälle sind nicht geeignet, den Heilwert des Serums zu beweisen. Zuviel Fälle kenne ich mit dem gleichen bakteriologischen $\mathrm{Be}$ fund, die in Heilung übergegangen sind, ohne daß Serum oder irgendeine besondere Therapie angewandt wäre.

Fußend auf diesen Erfahrungen, kann ich nich also nicht für berechtigt erachten, ein Untersuchungsverfahren anzuwenden, das an sich schon Schwerkranken offenkundigen Nachteil bringen kann. Denn nach dem, was wir heute über das $\mathrm{Zu}$ standekommen der puerperalen. Infektion wissen, ist es ja nicht auszuschließen, daß durch eine brüske Sekretentnahme der fortschreitenden Infektion Tür und Tor geöffnet wird. Und für diese Fälle ist es dann ein schlechter Trost zu sagen: Nun ja, die Streptococcen im Uterus haben die fortschreitende Entzündung verursacht, ihr Nachweis bringt mir die wissenschaftliche Erklärung des Falles. Der Sache selbst wird dadurch in lieiner Weise genutzt.

Man muß sich also immer vergegenwärtigen, daß die Verletzung des Endometriums vom dritten Tage an, die sich beim Einführen einer starren Röhre zur Sekretentnahme nicht immer vermeiden läht, zu einer Zeit erfolgt, in der sich der entzündliche Schutzwall an der Innenfläche des Uterus für die physiolo. gische Reaktion des Gewebes und der normale Schutz gegeniiber dem Tieferdringen der Bakterien bereits gebildet hat.

(Fortsetzung folgt.) 